\title{
Role of resistin in diet-induced hepatic insulin resistance
}

\author{
Evan D. Muse, ${ }^{1,2,3}$ Silvana Obici, ${ }^{2,3}$ Sanjay Bhanot, ${ }^{4}$ Brett P. Monia, ${ }^{4}$ Robert A. McKay, ${ }^{4}$ \\ Michael W. Rajala, ${ }^{5}$ Philipp E. Scherer, $2,3,5$ and Luciano Rossetti1,2,3
}

1Department of Molecular Pharmacology, 2Department of Medicine, and ${ }^{3}$ Diabetes Research and Training Center, Albert Einstein College of Medicine, New York, New York, USA. ${ }^{4}$ ISIS Pharmaceuticals, Carlsbad, California, USA. ${ }^{5}$ Department of Cell Biology, Albert Einstein College of Medicine, New York, New York, USA.

\begin{abstract}
Resistin is an adipose-derived hormone postulated to link adiposity to insulin resistance. To determine whether resistin plays a causative role in the development of diet-induced insulin resistance, we lowered circulating resistin levels in mice by use of a specific antisense oligodeoxynucleotide (ASO) directed against resistin mRNA and assessed in vivo insulin action by the insulin-clamp technique. After 3 weeks on a high-fat (HF) diet, mice displayed severe insulin resistance associated with an approximately $80 \%$ increase in plasma resistin levels. In particular, the rate of endogenous glucose production (GP) increased more than twofold compared with that in mice fed a standard chow. Treatment with the resistin ASO for 1 week normalized the plasma resistin levels and completely reversed the hepatic insulin resistance. Importantly, in this group of mice, the acute infusion of purified recombinant mouse resistin, designed to acutely elevate the levels of circulating resistin up to those observed in the HF-fed mice, was sufficient to reconstitute hepatic insulin resistance. These results provide strong support for a physiological role of resistin in the development of hepatic insulin resistance in this model.
\end{abstract}

\section{Introduction}

Epidemiological and metabolic evidence tightly links obesity to type 2 diabetes mellitus (DM2), and insulin resistance provides the strongest etiological thread (1-3). During the past decade, we have witnessed a parallel rise in the prevalence of obesity and DM2 among both children and adults $(4,5)$. This rapid epidemic is likely the consequence of multiple interactions between genes and environment. Consumption of high-calorie diets and sedentary lifestyles are deemed to be the main environmental triggers $(2,4)$. In this regard, an understanding of the mechanisms by which these environmental factors can lead to insulin resistance is particularly important. Adipose tissue is the main endogenous source of circulating lipids, but it is also the site of production and secretion of several hormones and cytokines. These adiposederived signaling molecules exert potent metabolic effects in distant organs, and they are likely to play a key role in the complex interorgan communication network, which appears to modulate intermediate metabolism and energy balance $(6,7)$. Resistin is an adipose-derived circulating protein and belongs to a new gene family of small cysteine-rich secreted proteins (8). Resistin (also known as ADSF and FIZZ3) has been postulated to participate in the regulation of glucose metabolism because its administration to rodents increased blood glucose levels (9) and hepatic glucose

\footnotetext{
Nonstandard abbreviations used: AMP-activated protein kinase (AMPK); antisense oligonucleotide (ASO); gluconeogenesis (GNG); glucose infusion rate (GIR); glucose-6-phosphatase (G6Pase); glycogen synthase kinase 3 (GSK3); high-fat (HF); intraperitoneal (i.p.); open reading frame (ORF); peroxisome proliferator-activated receptor- $\gamma$ (PPAR- $\gamma$ ); phosphoenolpyruvate (PEP); phosphoenolpyruvate carboxykinase (PEPCK); rate of endogenous glucose production (GP); rate of glucose appearance $(\mathrm{Ra})$; rate of glucose disappearance (Rd); standard chow (SC); triglyceride (TG); type 2 diabetes mellitus (DM2); uridinediphospho-glucose (UDP-glucose)

Conflict of interest: S. Bhanot, B.P. Monia, and R.A. McKay are employees of ISIS Pharmaceuticals, which has an interest in developing antisense therapeutics to treat diabetes and other diseases.

Citation for this article: J. Clin. Invest. 114:232-239 (2004).

doi:10.1172/JCI200421270
}

production (10). However, a physiological role of endogenous resistin in the development of diet-induced insulin resistance remains controversial, partly because of the lack of experimental evidence for the notion that resistin "loss-of-function" can exert meaningful effects on metabolic processes.

To determine if the elevation in circulating levels of resistin associated with high-fat (HF) feeding plays a significant role in the onset of insulin resistance, we tested whether lowering resistin concentrations in HF-fed mice is sufficient to improve in vivo insulin action. For this purpose, we employed a sequencespecific antisense oligodeoxynucleotide (ASO) in combination with infusion of recombinant resistin and with the insulinclamp technique in conscious mice.

\section{Results}

Treatment with resistin antisense normalized plasma resistin levels in HFfed mice. To determine the potential role of elevated circulating resistin levels in the development of diet-induced insulin resistance, we sought to lower the plasma resistin concentrations in HF-fed mice to the levels observed in standard chow-fed (SC-fed) mice. After an overnight fast, circulating levels of resistin as well as glucose and insulin were increased in HF-fed mice compared with SC-fed mice (Table 1). Treatment of HF-fed mice with resistin ASO markedly lowered the plasma concentrations of resistin, glucose, and insulin to levels that were indistinguishable from those of SC-fed mice (Table 1). In a separate cohort of mice, we also measured the plasma resistin levels after short-term withdrawal of food ( $\sim 5 \mathrm{~h})$ (Figure 1). Once again, plasma resistin levels were markedly increased by HF feeding $(77.0 \pm 11.5$ vs. $42.2 \pm 5.1 \mathrm{ng} / \mathrm{ml})$ when control ASOs were injected (Figure 1C; HF + ConASO vs. $\mathrm{SC}+\mathrm{ConASO}$; Con, control). However, treatment with resistin ASO restored the circulating resistin levels $(46.1 \pm 4.8 \mathrm{ng} / \mathrm{ml})$ to those observed in mice on SC. Importantly, in the HF + RsASO + i.v. Rs (Rs, resistin) group, the acute infusion of recombinant mouse resistin elevated the plasma resistin levels $(73.0 \pm 5.0$ 
Table 1

Effect of resistin ASO on the circulating fasting levels of glucose, insulin, leptin, resistin, and adiponectin

$\begin{array}{lccc}\text { Group } & \begin{array}{c}\mathbf{1} \\ \text { (SC + ConASO) }\end{array} & \begin{array}{c}\mathbf{2} \\ (\mathbf{H F}+\text { ConASO) }\end{array} & \begin{array}{c}\mathbf{3} \\ (\mathbf{H F}+\mathbf{R s A S 0})\end{array} \\ \text { Glucose }(\mathrm{mg} / \mathrm{dl}) & 141 \pm 7 & 169 \pm 5 & 111 \pm 11^{\mathrm{A}} \\ \text { Insulin }(\mathrm{ng} / \mathrm{ml}) & 0.30 \pm 0.02 & 0.44 \pm 0.05^{\mathrm{B}} & 0.23 \pm 0.06^{\mathrm{A}} \\ \text { Leptin }(\mathrm{ng} / \mathrm{ml}) & 0.28 \pm 0.04 & 0.37 \pm 0.05 & 0.38 \pm 0.04 \\ \text { Resistin }(\mathrm{ng} / \mathrm{ml}) & 105 \pm 9 & 160 \pm 11^{\mathrm{B}} & 111 \pm 14^{\mathrm{A}} \\ \text { Adiponectin }(\mu \mathrm{g} / \mathrm{ml}) & 7.18 \pm 0.37 & 6.37 \pm 0.68 & 6.49 \pm 0.46\end{array}$

${ }^{A} P<0.05$ vs. group $2(\mathrm{HF}+\mathrm{ConASO}) ;{ }^{B} P<0.05$ vs. group 1 (SC + ConASO).

$\mathrm{ng} / \mathrm{ml}$ ) to levels observed in the HF + ConASO group. Average body weight was modestly lower in the mice that received SC $(27.6 \pm 0.3 \mathrm{~g})$ compared with the mice that received HF $(28.9 \pm 0.5$, $28.2 \pm 0.3$, and $28.7 \pm 0.4 \mathrm{~g}$ in HF + ConASO, HF + RsASO, and $\mathrm{HF}+\mathrm{RsASO}+$ i.v. Rs groups, respectively). No significant differences occurred in the mean body weights among the three groups of mice on HF. After a 5-hour fast (postabsorptive state), the plasma glucose concentrations were significantly higher in the HF groups $(154 \pm 7,146 \pm 5$, and $155 \pm 2 \mathrm{mg} / \mathrm{dl}) \mathrm{com}-$ pared with the SC group $(129 \pm 4 \mathrm{mg} / \mathrm{dl})$. Similarly, the hepatic triglyceride (TG) content at the completion of the infusion studies was markedly higher in the HF groups compared with the SC group (Figure 1D). However, no significant differences occurred in the plasma glucose and hepatic TG levels among the three groups of mice on HF. No significant differences occurred in the mean plasma insulin levels during the clamp studies (Figure 1B).

Effect of resistin ASO on glucose disposal and production. To examine the role of resistin in diet-induced insulin resistance, we assessed the metabolic effects of insulin in the four experimental groups (Figure 2). Similar moderate increases in the plasma insulin concentrations were generated in conscious mice, and the plasma glucose concentrations were maintained at approximately $8 \mathrm{mM}$ by a variable glucose infusion. Thus, the effects of physiological changes in the circulating resistin levels on glucose kinetics were assessed in the presence of similar steady-state insulin and glucose levels. To acutely restore the circulating resistin levels to those observed in HF-fed mice that received control ASO (HF + ConASO), 6 HF-fed mice treated with resistin ASO received a primed-constant infusion of recombinant mouse resistin (HF + RsASO + i.v. Rs), whereas all other groups received a similar infusion of vehicle (saline) (Figure 2A).
We first examined the effect of increased circulating insulin concentrations on the glucose infusion rate (GIR) and tissue rate of glucose disappearance (Rd), or uptake (Figure 2, B and C). During this period, insulin levels were similarly elevated in all groups (3.6 $\mathrm{mU} / \mathrm{kg} / \mathrm{min}$ ). Importantly, all measurements were performed during the final 50 minutes of the 90-minute hyperinsulinemic-clamp study, a time after steady-state conditions for plasma glucose and insulin concentrations, glucose-specific activity, and rates of glucose infusion were achieved. The rates of exogenous glucose infusion required to maintain the target plasma glucose concentration during the insulin clamp were decreased by approximately $59 \%$ in HF-fed mice compared with SC-fed mice. This marked decrease in GIR was caused by a moderate decrease in $\mathrm{Rd}(P=0.038 \mathrm{HF}$ vs. $\mathrm{SC}$; Figure $2 \mathrm{~B}$ ) and to a marked increase in the rate of endogenous glucose production (GP) (Figure 2C). A 1-week treatment with resistin ASO failed to alter Rd (Figure $2 \mathrm{~B}$ ). However, normalizing the plasma resistin levels in HF-fed mice completely restored GP to levels observed in the SC group (Figure 2C). The acute infusion of recombinant resistin did not significantly alter the Rd. By contrast, GP was markedly higher during the infusion of resistin than during vehicle infusion (Figure $2 \mathrm{C}$ ), and it was now similar to that measured in HF-fed mice treated with control ASO. These
A



C

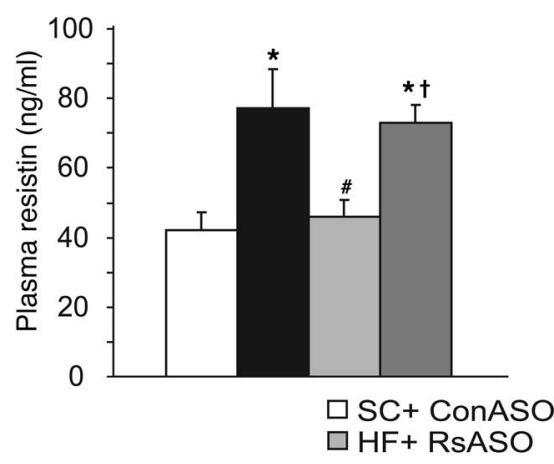

Figure 1

Treatment with resistin ASO normalized plasma resistin levels in HF-fed mice. (A) Experimental design for treatment with resistin ASO and clamp studies. Mice on HF diet for 3 weeks received an i.p. injection of resistin ASO (RsASO) or control ASO (ConASO) 7 and 3 days before the insulin-clamp studies. Intravenous catheters were inserted into the jugular vein 3 days before the clamp procedure. (B) Plasma insulin levels at the end of insulin clamp. Insulin levels were similar in all experimental groups. (C) Plasma resistin levels. Circulating resistin levels are significantly increased in mice on HF diet (black bar) as compared with mice on SC (white bar). Treatment with resistin ASO significantly decreased circulating resistin levels to those of SC-fed mice. Finally, infusion of recombinant mouse resistin acutely restored circulating resistin levels (HF + RsASO + i.v. Rs) to those observed in the HF-fed mice treated with control ASO. (D) Increased liver TGs with HF diet. Hepatic TG content was increased twofold by HF diet, whereas treatment with resistin ASO or acute infusion of recombinant resistin did not significantly altered hepatic TG levels. ${ }^{*} P<0.01$ vs. SC group; ${ }^{*} P<0.01$ vs. HF + ConASO; † $P<0.01$ vs. HF + RsASO. ww, wet weight. 
A

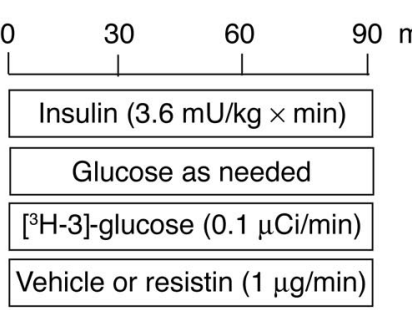

C

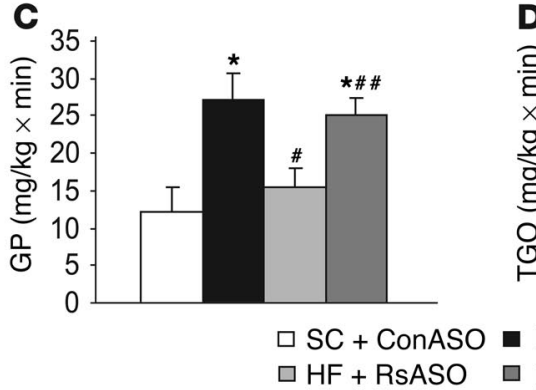

B

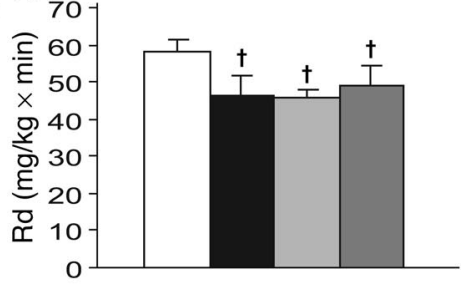

\section{D}

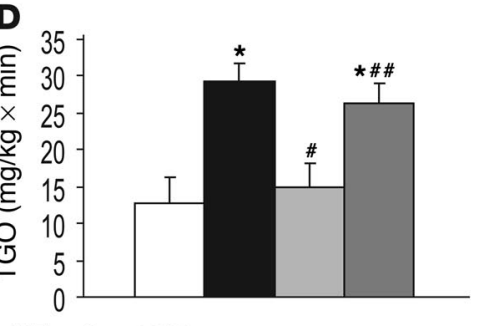

\section{Figure 2}

Circulating resistin is required for diet-induced hepatic insulin resistance. (A) Schematic representation of the insulin-clamp procedure. The infusion studies lasted a total of 90 minutes. Mice received a primed-constant infusion of HPLC-purified [ $\left.{ }^{3} \mathrm{H}-3\right]$-glucose $(0.1 \mu \mathrm{Ci} / \mathrm{min})$ and insulin $(3.6 \mathrm{mU} / \mathrm{kg} / \mathrm{min})$ at $t=0$ minutes for the duration of the study. A variable infusion of a $10 \%$ glucose solution was started and periodically adjusted (glucose as needed) to maintain the plasma glucose concentration at approximately $8 \mathrm{mM}$ for the rest of the study. (B) Rates of glucose uptake (Rd) during the insulin-clamp studies. (C) Rates of endogenous glucose production (GP) during the insulinclamp studies. (D) Effect of resistin ASO and resistin infusion on total glucose output (in vivo flux-through G6Pase). ${ }^{\dagger} P<0.05$ vs. SC group; ${ }^{*} P<0.01$ vs. SC group; $\# P<0.01$ vs. HF + ConASO; \# $P<0.01$ vs. HF + RsASO. results indicate that short-term intravenous infusion of resistin stimulates glucose production in the presence of moderately elevated hyperinsulinemia. Perhaps more importantly, bidirectional changes in plasma resistin concentrations have a major impact on the regulation of hepatic glucose production, and these effects are likely to play a pivotal role early in the development of hepatic insulin resistance during high-fat feeding.

Effect of resistin ASO on hepatic glucose fluxes. GP represents the net contribution of glucosyl units derived from gluconeogenesis and glycogenolysis. However, a portion of glucose entering the liver via phosphorylation of plasma glucose is also a substrate for dephosphorylation via glucose-6-phosphatase (G6Pase). This futile cycle between glucokinase and G6Pase is commonly called glucose cycling and accounts for the difference between the total glucose output (flux-through G6Pase) and GP.

To further define the mechanisms by which resistin modulates hepatic glucose production, we estimated the in vivo flux-through G6Pase (Figure 2D) and the relative contribution of glucose cycling, gluconeogenesis, and glycogenolysis to glucose output (Table 2 and Figure 3 ) in a subgroup of animals. Table 2 displays the specific activities of $\left[{ }^{14} \mathrm{C}\right]$-phosphoenolpyruvate $\left(\left[{ }^{14} \mathrm{C}\right]-\mathrm{PEP}\right),\left[{ }^{3} \mathrm{H}\right]$-urinediphospho-glucose $\left(\left[{ }^{3} \mathrm{H}\right]-U D P-\right.$ glucose), $\left[{ }^{14} \mathrm{C}\right]$-UDP-glucose, and $\left[{ }^{3} \mathrm{H}-3\right]$-glucose used to calculate the contribution of PEP and plasma glucose (Indirect and Direct in Table 2) to the hepatic glucose-6phosphate pool. These data allowed us to estimate the in vivo fluxes through G6Pase, the rates of glucose cycling, PEP-gluconeogenesis, and glycogenolysis in all groups. In the presence of similar plasma insulin concentra- tions, the rate of glucose production (shown in Figure 2) was decreased by resistin ASO and increased by resistin infusion. As shown in Figure 2D, resistin ASO markedly decreased and resistin infusion markedly increased the flux-through G6Pase in parallel to their effects on GP. Consistent with this marked changes in overall glucose output, the rates of glucose cycling were also decreased in mice treated with resistin ASO and increased by the acute infusion of recombinant resistin (Figure $3 \mathrm{~A}$ ). Thus, bidirectional changes in the circulating levels of the adiposederived protein resistin lead to marked changes in the in vivo flux-through G6Pase. The latter effects were accounted for by marked and parallel changes in the rates of both gluconeogenesis and glycogen breakdown (Figure 3, A and B).

Effect of resistin ASO on hepatic phosphoenolpyruvate carboxykinase and G6Pase $m R N A$. On the basis of the above flux data, we explored two potential molecular targets of resistin action in the liver. G6Pase and phosphoenolpyruvate carboxykinase (PEPCK) are important determinants of hepatic glucose fluxes, and their regulation by insulin involves transcriptional events. Thus, we assessed the

\section{Table 2}

Effect of resistin ASO and resistin infusion on the "direct" and "indirect" pathway of hepatic UDPglucose formation

\begin{tabular}{|c|c|c|c|c|}
\hline Group & $\begin{array}{c}1 \\
\text { (SC + ConASO) }\end{array}$ & $\begin{array}{c}2 \\
(H F+\text { ConASO })\end{array}$ & $\begin{array}{c}3 \\
\text { (HF+RsASO) }\end{array}$ & $\begin{array}{c}4 \\
\text { (HF+RsASO+Rs) }\end{array}$ \\
\hline$\left[{ }^{3} \mathrm{H}\right]$-UDP-glucose liver SA (dpm/nmol) & $1.1 \pm 0.7$ & $1.9 \pm 0.5$ & $1.4 \pm 0.6$ & $1.3 \pm 0.5$ \\
\hline$\left[{ }^{3} \mathrm{H}-3\right]$-Glucose plasma SA (dpm/nmol) & $19.5 \pm 2.0$ & $24.9 \pm 1.4$ & $26.8 \pm 1.5$ & $25.4 \pm 1.3$ \\
\hline$\%$ Direct & $5.1 \pm 1.0$ & $7.6 \pm 1.1$ & $4.9 \pm 0.7$ & $5.0 \pm 0.6$ \\
\hline$\left[{ }^{14} \mathrm{C}\right]$-UDP-glucose liver SA (dpm/nmol) & $2.1 \pm 0.9$ & $2.3 \pm 0.6$ & $1.9 \pm 0.8$ & $2.2 \pm 1.2$ \\
\hline$\left[{ }^{14} \mathrm{C}\right]$-PEP liver SA (dpm/nmol) & $8.7 \pm 1.2$ & $6.7 \pm 1.6$ & $6.4 \pm 1.8$ & $6.8 \pm 0.5$ \\
\hline$\%$ Indirect & $12.8 \pm 3.8$ & $17.0 \pm 4.4$ & $14.8 \pm 2.3$ & $15.7 \pm 3.8$ \\
\hline
\end{tabular}

Specific activities of plasma glucose, hepatic UDP-glucose (UDP-GIc), and phosphoenolpyruvate (PEP) were used to calculate the contribution of plasma glucose and PEP-gluconeogenesis to the hepatic UDP-glucose pool after [ $\left.{ }^{3} \mathrm{H}-3\right]$-glucose and $\left[\mathrm{U}-{ }^{14} \mathrm{C}\right]$-lactate infusions in rats at the completion of pancreatic/insulin-clamp studies. $\%$ Direct, percent of the hepatic UDP-glucose pool derived from plasma glucose calculated as the ratio of the spe-

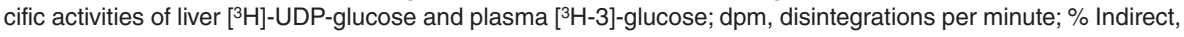
percent of the hepatic UDP-glucose pool derived from PEP-gluconeogenesis, calculated as the ratio of the specific activities of $\left[{ }^{14} \mathrm{C}\right]$-UDP-glucose and $2 \times\left[{ }^{14} \mathrm{C}\right]$-PEP; SA, specific activity. 


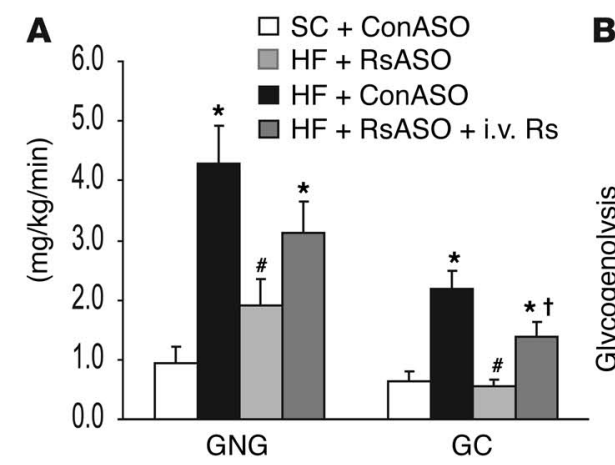

B
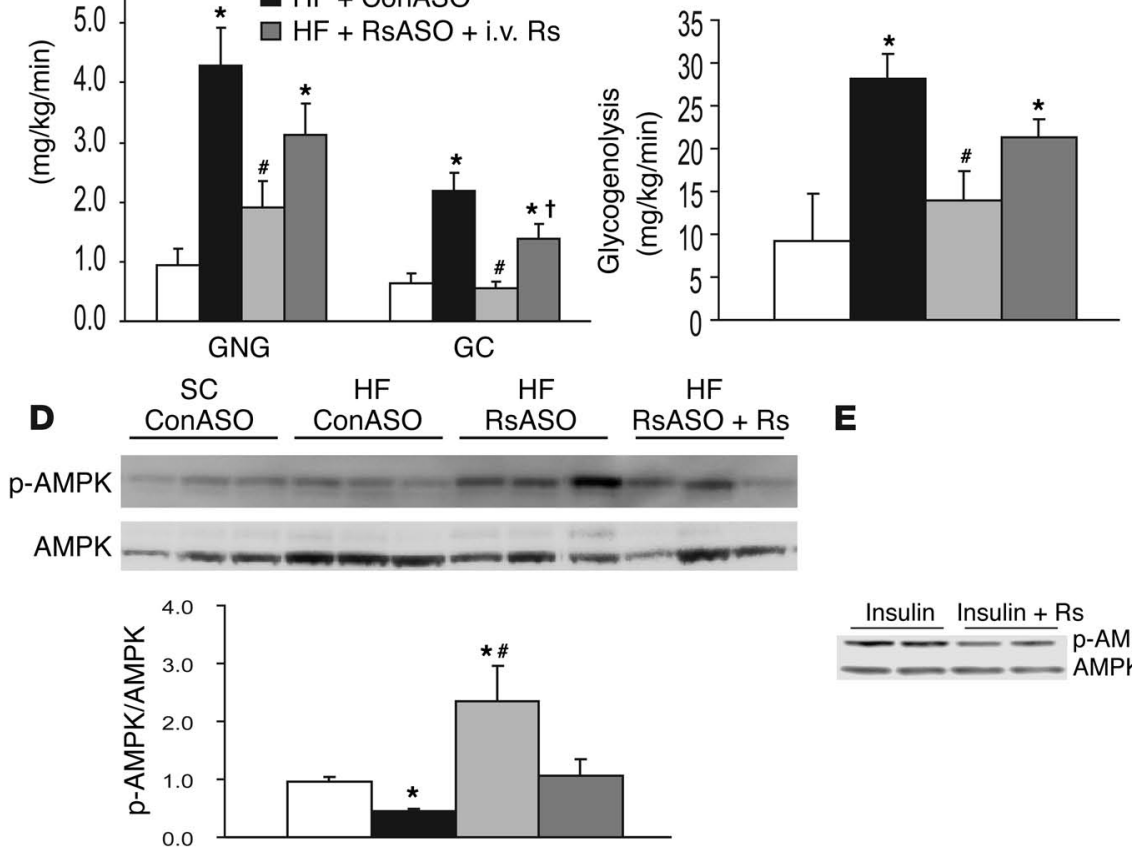

E

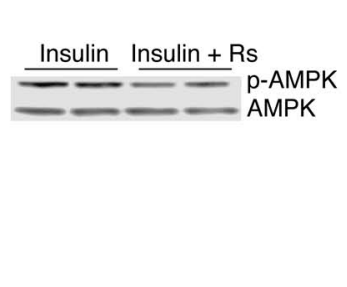

C

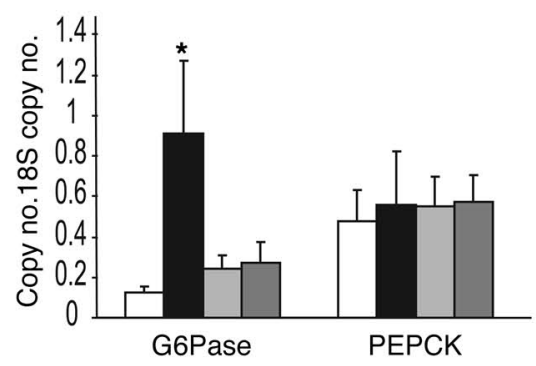

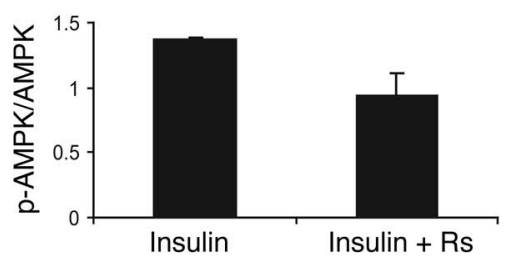

Figure 3

Effect of resistin antisense and resistin infusion on hepatic glucose fluxes during insulin-clamp studies. (A) Effect of resistin antisense and resistin infusion on the rates of PEP-gluconeogenesis (GNG) and glucose cycling (GC). (B) Effect of resistin antisense and resistin infusion on the rate of glycogenolysis. (C) Effect of resistin antisense and resistin infusion on hepatic G6Pase and PEPCK mRNA. (D) Effect of resistin antisense and resistin infusion on hepatic AMPK phosphorylation (p-AMPK). (E) Effect of recombinant resistin on AMPK phosphorylation in isolated primary hepatocytes. ${ }^{*} P<0.01$ vs. SC group; ${ }^{~} P<0.01$ vs. HF + ConASO; ${ }^{\dagger} P<0.01$ vs. HF + RsASO.

effect of resistin ASO and resistin infusion on G6Pase and PEPCK gene expression in liver harvested at the completion of the clamp studies. Real-time PCR analyses revealed that HF feeding resulted in a marked increase in liver G6Pase mRNA levels. Importantly, normalization of plasma resistin levels in HF-fed rats markedly decreased liver G6Pase mRNA abundance at levels that were similar to those of SC-fed rats (Figure 3C). However, neither HF feeding nor changes in circulating resistin resulted in significant changes in PEPCK mRNA in the liver (Figure 3C). The acute infusion of recombinant resistin failed to significantly alter the hepatic expression of these gluconeogenic enzymes.

Effect of resistin ASO on hepatic AMP kinase phosphorylation. We examined the abundance of phosphorylated AMP-activated protein kinase (AMPK) in liver by Western blot analysis (Figure 3D). HF feeding significantly decreased phospho-AMPK. Treatment with resistin ASO resulted in a significant increase in the phosphorylation of AMPK in the liver, and the infusion of recombinant resistin largely reversed this effect. The incubation of

\section{Figure 4}

Effect of resistin antisense on phosphorylation of hepatic Akt and GSK3. (A) Experimental design for treatment with resistin ASO and acute stimulation with insulin (100 mU). (B) Effect of resistin ASO on phosphorylation of Akt on serine 473 (p-Akt473) and GSK3 (p-GSK3) in liver extracts from HF-fed mice treated with ConASO and RsASO. Unstimulated samples, saline alone, are included as negative controls. ${ }^{*} P<0.05$ vs. HF + ConASO group. primary rat hepatocytes with recombinant resistin moderately decreased AMPK phosphorylation (Figure 3E).

Effect of resistin ASO on hepatic Akt and glycogen synthase kinase 3 phosphorylation. To examine potential effects of "hyper-resistinemia" on liver insulin signaling, we injected fasted mice intraperitoneally (i.p.) with a bolus of insulin and sampled the liver 15 minutes later
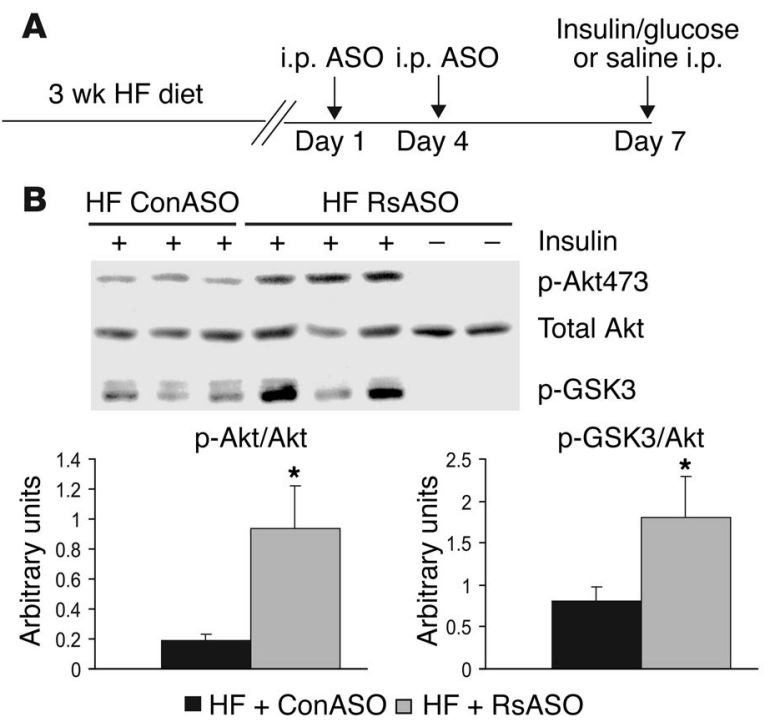
(Figure 4A). The abundance of phosphorylated and total Akt and phosphorylated glycogen synthase kinase 3 (GSK3) were assessed in liver by Western blot analysis (Figure 4B). Acute administration of insulin did not alter total Akt but significantly enhanced Akt and GSK3 phosphorylation. Treatment of HF-fed mice with resistin ASO resulted in a significant increase in the phosphorylation of both Akt and GSK3 in the liver.

\section{Discussion}

Diet-induced insulin resistance is a relevant model for the most common forms of insulin resistance in humans. In this regard, the onset of hepatic insulin resistance typically precedes the appearance of peripheral insulin resistance in human (11) and animal $(12,13)$ models of voluntary overfeeding. However, the molecular basis responsible for this rapid metabolic adaptation remains elusive. Increased flux of free fatty acids rapidly induces hepatic and peripheral insulin resistance, and, thus, diet-induced changes in lipid fluxes may play a significant role in the development of this form of insulin resistance (14-16). However, adipose tissue is also an active endocrine organ that secretes numerous circulating proteins, some with potent effects on energy and intermediary metabolism and on insulin signaling (9, 17-19). Consistent with this postulate, the insulin-sensitizing effects of peroxisome proliferator-activated receptor- $\gamma$ (PPAR- $\gamma$ ) agonists (20) may be partly caused by the regulation of the biosynthesis and secretion of adipose-derived proteins such as resistin (9, 21, 22) and Acrp30/ adiponectin (23). Of interest, resistin is expressed at higher levels in intra-abdominal than subcutaneous fat depots in human (24). Most important, the infusion of recombinant resistin has been shown to increase plasma glucose levels and to stimulate endogenous glucose production (10) in rodents, and plasma resistin levels are significantly increased in mice fed an HF diet compared with a standard low-fat/high-carbohydrate diet (25).

Is the increase in circulating resistin levels partly responsible for the development of insulin resistance? To address this question, we sought to reverse the diet-induced increase in circulating resistin levels to assess its impact on insulin action and glucose fluxes. To this end, we used a sequence-specific ASO that targets the resistin gene. Indeed, treatment with resistin ASO lowered the plasma resistin levels in HF-fed mice to the levels observed in SC-fed mice. Because food intake and body weight were similar in all HF-fed mice, this experimental approach allowed us to isolate the contribution of hyper-resistinemia to the metabolic alteration induced by high-fat feeding. Indeed, normalization of the circulating resistin concentration per se markedly improved hepatic insulin action in HF-fed mice. This effect was sufficient to completely reverse the increase in GP induced by high-fat feeding. On the other hand, the decrease in the plasma resistin levels failed to modify the rate of glucose uptake. Thus, the elevation in circulating resistin levels induced by high-fat feeding plays an important role in the etiology of hepatic but not of peripheral insulin resistance in this animal model. To verify the specificity of the metabolic changes induced by lowering the circulating resistin levels, we performed a reconstitution experiment in which we acutely restored the hyper-resistinemia induced by high-fat feeding in HF-fed mice treated with resistin ASO. The infusion of recombinant mouse resistin elevated the plasma resistin concentration to the levels measured in HF-fed mice treated with control ASO. This protocol was sufficient to reproduce the hepatic insulin resistance induced by high-fat feeding. Thus, bidirectional and physiological changes in the circulat- ing resistin concentrations have a major impact on hepatic glucose output, and they are likely to largely account for the development of hepatic insulin resistance in this mouse model of diet-induced insulin resistance. Although this model of diet-induced insulin resistance displays several of the typical features, such as moderate fasting hyperglycemia and hyperinsulinemia and hepatic and peripheral insulin resistance, one should use caution in passively extrapolating the present findings to other insulin-resistant states. Of note, we had previously reported that infusion of recombinant mouse resistin in rats increased the plasma resistin levels to approximately $200 \mathrm{ng} / \mathrm{ml}$ and induced hepatic insulin resistance (10). The present study provides the first evidence that changes in the circulating resistin levels within a physiological range (40-80 $\mathrm{ng} / \mathrm{ml}$ ) can also modulate glucose production in the presence of moderately elevated insulin levels. Conversely, we failed to demonstrate significant effects of these modest 1 -week changes in plasma resistin levels on peripheral insulin action. It is entirely possible that larger or more prolonged changes in circulating resistin levels may antagonize insulin action in extrahepatic sites.

To investigate the mechanisms by which resistin increases the net production of glucose by the liver, we estimated the overall rate of glucose output (in vivo flux through G6Pase) and the relative contribution of hepatic glucose phosphorylation (glucose cycling), gluconeogenesis, and glycogenolysis to this flux. Our assessment of G6Pase flux revealed an approximately threefold increase with HF feeding compared with SC feeding. This increase closely paralleled that seen with net glucose production, which suggests that activation of G6Pase by resistin is a likely mechanism of action. Of interest, the marked increases in all pathways involved in the formation of liver glucose-6-phosphate (gluconeogenesis, glycogenolysis, and glucose cycling) induced by resistin infusion are also consistent with the increased flux through G6Pase being the driving force for the enhanced rate of glucose output. The marked increase in the in vivo flux through G6Pase in the HF group may be partly ascribed to increased hepatic expression of the catalytic unit of G6Pase. The latter increase was largely reversed by 1 -week normalization of the plasma resistin levels. However, short-term increase in plasma resistin levels markedly decreased total glucose output but did not significantly change the liver G6Pase mRNA levels. Thus, the potent effects of resistin on total glucose output are likely to involve both transcriptional and posttranscriptional events. Because liver AMPK is required for the effects of adiponectin on plasma glucose levels (26), and its activation decreases G6Pase expression (27), we investigated whether changes in circulating resistin levels alter AMPK phosphorylation in the liver. Indeed, decreasing the plasma resistin concentration by ASO treatment increased AMPK phosphorylation, and the infusion of recombinant resistin decreased this parameter. These findings suggest that one of the hepatic mechanisms of action of adipose-derived hormones such as adiponectin $(26,28)$, leptin $(29)$, and resistin converge on this fuel-sensing enzyme. Conversely, the acute infusion of resistin rapidly decreased AMPK phosphorylation in the liver but did not alter G6Pase expression. Thus, if changes in AMPK contribute to the resistin-induced changes in G6Pase expression, a lag time appears to occur between these effects. Furthermore, the increased flux through G6Pase in response to resistin infusions did not require changes in mRNA levels. Overall, whether the changes in hepatic AMPK activity are responsible for the rapid effects of resistin on GP remains to be elucidated. In fact, a decrease in AMPK activity should decrease fat oxidation via elevations in malonyl-CoA lev- 
els, and a decrease in fat oxidation would, in turn, be expected to decrease rather than increase gluconeogenesis.

In humans, resistin is expressed at low levels in adipose cells and at much higher levels in macrophages $(25,30)$. However, plasma resistin levels are elevated in human obesity, and some studies have reported a positive correlation of plasma resistin with obesity and insulin resistance in humans $(31,32)$. Importantly, antidiabetic drugs acting via stimulation of PPAR- $\gamma$ receptors reduce resistin expression in both human macrophages (30) and rodent adipose cells $(33,34)$. Taken together with the emerging role of macrophages in the metabolic syndrome and in obesity $(25,35)$, these observations suggest that resistin may exert important metabolic functions in both rodents and humans, despite the divergent tissue pattern of expression.

The identification of an important role of endogenous resistin in the development of diet-induced insulin resistance in mice raises the possibility that this novel adipose/macrophage-derived hormone in concert with other signaling molecules plays a pivotal role in the association of obesity and hepatic insulin resistance in humans.

\section{Methods}

Animals. Adult male C57BL6J mice (27-32 g) were anesthetized with chloral hydrate $(400 \mathrm{mg} / \mathrm{kg}$ bw i.p.) and catheterized through the right internal jugular vein as previously described (36). The venous catheter was used for infusion, and blood samples were collected from the tail vein. Each animal was monitored for food intake and weight gain after surgery to ensure complete recovery. Thirty-two mice were randomized into four experimental groups. During the 3 weeks preceding the clamp study, one group received SC (Lab Diet; Purina Mills International, St. Louis, Missouri, USA) from which $59 \%$ of calories were provided by carbohydrate, $28 \%$ were provided by protein, and $12 \%$ were provided by fat. The remaining three groups received HF chow (Purina Mills International) from which $45 \%$ of calories were provided by carbohydrate, $22 \%$ were provided by protein, and $33 \%$ were provided by fat). Thus, we studied four groups of mice: group 1 consisted of SC-fed mice treated with control ASO, group 2 consisted of HF-fed mice treated with control ASO, group 3 consisted of HF-fed mice treated with resistin ASO, and group 4 consisted of HF-fed mice treated with resistin ASO and acutely infused with recombinant mouse resistin. All mice received two i.p. injections $(25 \mathrm{mg} / \mathrm{kg}$ ) of either control ASO (groups 1 and 2) or resistin ASO (groups 3 and 4) during the week preceding the clamp study (Figure 1A). For insulin tolerance testing, basal plasma values and hepatic kinase phosphorylation studies, adult male C57BL6J mice were fed SC and HF diets and treated with control and resistin ASO as described above. After an overnight fast, tail blood was sampled for serum glucose and hormone analysis, and animals were injected i.p. with $100 \mathrm{mU}$ insulin (human recombinant; Sigma-Aldrich, St. Louis, Missouri, USA) in a solution of $5 \%$ glucose (Sigma-Aldrich) in normal saline. After 15 minutes, animals were sacrificed and livers and intracardial blood were sampled.

Cell culture. Primary rat hepatocytes were obtained from the Cell Culture and Genetic Engineering Core Facility of the Marion Bessin Liver Research Center of the Albert Einstein College of Medicine (37). After cell attachment to the culture plate growth media was changed to DMEM (Invitrogen, Carlsbad, California, USA) $+10 \%$ FBS (Invitrogen) with either insulin (10 ng/ml; SigmaAldrich) or insulin plus recombinant resistin $(1 \mu \mathrm{g} / \mathrm{ml})$. Cell lysates were prepared after an overnight incubation and analyzed by Western blot as described below.
Design of oligodeoxynucleotide antisense against resistin $m R N A$. The antisense oligodeoxynucleotide (ODN), Res-AS (ISIS Inc., Carlsbad, California, USA), was designed to hybridize to the sequence-spanning mouse resistin mRNA. All nucleotides were synthesized as uniform phosphothiorate chimeric ODNs, with 2'-O-methoxyethyl (MOE) groups on bases 1 to 5 and 16 to 20 . The ODN were synthesized on an Applied Biosystems 380B automated DNA synthesizer (PerkinElmer-Applied Biosystems, Boston, Massachusetts, USA) and purified as described (38). Mouse resistin ASO (ISIS $167308)$ is a 20-base, 5-10-5 MOE chimeric ASO with the following sequence: TTCACGAATGTCCCACGAGC. It hybridizes to position 331 on the mouse resistin sequence (GenBank accession number AF323080.1). The control ASO (ISIS 29848) is a chemistry control ASO that has the same length and chemical makeup as the resistin ASO but is composed of all 419 possible ASO combinations when each base position is randomly synthesized with any of the four possible nucleotides (A, G, T, or C). Thus, it is not expected to hybridize to any mRNA sequence.

Primers and real-time PCR. Liver G6Pase and PEPCK mRNAs were measured by quantitative PCR with the following mouse primers: forward primer 5'-TCCTGGGACAGACACACAAG-3' and reverse primer $5^{\prime}$-CAACTTTAATATACGCTATTGG-3' for G6Pase; forward primer $5^{\prime}$-CTTCTCTGCCAAGGTCATCC-3' and reverse primer $5^{\prime}$-TTTTGGGGATGGGCAC-3' for PEPCK. The mRNA levels for G6Pase and PEPCK were normalized to $18 \mathrm{~S}$ expression (forward primer 5'-AGGGTTCGATTCCGGAGAGG-3', reverse primer 5'-CAACTTTAATATACGCTATTGG-3'). Total RNA was isolated with Trizol (Invitrogen) and single-strand cDNA was synthesized by application of Superscript (Invitrogen). Real-time PCR reactions were prepared with a LightCycler reaction kit (Roche, Indianapolis, Indiana, USA). A real-time PCR reaction of $20 \mu \mathrm{l}$ contained primers $(200 \mathrm{nM})$, reaction buffer, $2.3 \mathrm{mM} \mathrm{MgC}_{12}$, SYBR Green $2 \mu \mathrm{l}, 2 \mu \mathrm{l}$ of cDNA, and Taq DNA polymerase. Routinely, the reactions were cycled approximately 40 times. The reactions were carried out in capillaries on a LightCycler instrument (Roche).

Recombinant resistin. The entire open reading frame (ORF) of resistin were cloned into pFM1, which contains an internal ribosome entry site followed by the ORF of GFP as previously described (19). Stably transfected HEK 293-T cells were sorted by FACS for high levels of GFP expression and, therefore, high resistin expression. Five sorts of enrichment established a line of cells capable of producing milligram quantities of recombinant protein per liter of media. Serum-free media was used to collect the secreted protein from confluent cells for 2 days. The media was then harvested, spun down to remove cells and the $\mathrm{pH}$ adjusted to $\mathrm{pH} 6.0$ with $20 \mathrm{mM}$ Bis-Tris $\mathrm{pH}$ 6.0 . The media was then filtered through a $0.22-\mu \mathrm{m}$ filter and loaded directly onto an Econo-S ion exchange column (Bio-Rad Laboratories Inc., Hercules, California, USA). The protein was then eluted with a step salt gradient from $200 \mathrm{mM}$ to $300 \mathrm{mM} \mathrm{NaCl}$, followed by a $300 \mathrm{mM}$ to $1 \mathrm{M} \mathrm{NaCl}$ continuous gradient. Two major peaks were apparent, with resistin eluting in the second peak between approximately 350 and $500 \mathrm{mM} \mathrm{NaCl}$. This eluate was then concentrated by centrifugation in a centricon YM-3 (Millipore, Bedford, Massachusetts, USA) and loaded onto a Superdex 200 size filtration column (Amersham-Pharmacia, Piscataway, New Jersey, USA). The major peak contained at least $99 \%$ pure recombinant protein.

Measurement of mouse resistin, adiponectin, leptin, and insulin protein levels in plasma. Plasma mouse resistin and adiponectin levels from clamped animals were measured by RIA (Linco Inc., Austin, Texas, USA), whereas serum resistin, insulin, and leptin levels from fasted 
mice that received an acute bolus of insulin/glucose solution were measured via immunoassay that utilized Lincoplex microspheres analyzed by a Luminex-100 (Linco Inc.). RIA was also used for the measurement of adiponectin in fasted mice.

Measurements of in vivo glucose kinetics and insulin-clamp procedures. Euglycemic clamps were performed in conscious, unrestrained, catheterized mice as previously described $(28,36)$. Food was removed for 5 hours before beginning of in vivo studies. The infusion studies lasted a total of 90 minutes. Briefly, at 0 minutes (time $[t]=0$ minutes $)$, a primed $(2.5 \mu \mathrm{g})$, continuous $(1 \mu \mathrm{g} / \mathrm{h})$ i.v. infusion of resistin (group 4) or vehicle (group 1 to group 3) was initiated and maintained for the remainder of the study. The total dose of resistin infused into group 4 was $4 \mu \mathrm{g} /$ mouse. A solution of glucose $(10 \% \mathrm{wt} / \mathrm{vol})$ was infused at a variable rate as required to maintain euglycemia $(8 \mathrm{mM})$. Mice received a constant infusion of HPLC-purified [ $\left.{ }^{3} \mathrm{H}-3\right]$-glucose $(0.1 \mu \mathrm{Ci} / \mathrm{min}$; New England Nuclear, Boston, Massachusetts, USA), and insulin (3.6 $\mathrm{mU} / \mathrm{min} / \mathrm{kg} \mathrm{bw})$. Thereafter, plasma samples were collected to determine glucose levels (at $t=10,20,30,40,50,60,70,80$, and 90 minutes) as well as $\left[{ }^{3} \mathrm{H}\right.$-3]-glucose-specific activity (at $t=40,50,60,70,80$, and 90 minutes). Additionally, in 5 mice from each experimental group, 10 minutes before the end of the in vivo studies, $\left[\mathrm{U}-{ }^{14} \mathrm{C}\right]$-lactate (5- $\mu \mathrm{Ci}$ bolus, $0.4 \mu \mathrm{Ci} / \mathrm{min}$; New England Nuclear) was administered to determine the contribution of gluconeogenesis to the hepatic glucose-6-phosphate pool. Consecutive samples were pooled together for the assessment of the plasma insulin and resistin levels. Steady state conditions for both plasma glucose concentration and specific activity were achieved by 40 minutes in these studies. At the end of the in vivo studies, mice were anesthetized (pentobarbital $60 \mathrm{mg} / \mathrm{kg}$ i.v.), the abdomen was quickly opened and adipose tissue and liver were freeze-clamped in situ with aluminum tongs that were cooled in liquid nitrogen. The time between the injection of anesthesia and the freeze clamping of tissue samples was less than 60 seconds. Tissue samples were stored at $-80^{\circ} \mathrm{C}$ for further analysis.

Analytical procedures. Liver triglycerides were measured as described. Plasma glucose was measured by the glucose oxidase method on a Glucose Analyzer II (Beckman Instruments Inc., Fullerton, California, USA). Under steady-state conditions for plasma glucose concentration, the glucose Rd equals the rate of glucose appearance $(\mathrm{Ra})$. Ra was determined from the ratio of the infusion rate for $\left[{ }^{3} \mathrm{H}-3\right]$-glucose (disintegrations per minute) and the specific activity of plasma $\left[{ }^{3} \mathrm{H}-3\right]$-glucose (disintegrations per minute per milligram glucose) under steady-state conditions. The rate of GP was, therefore, obtained from the difference between $\mathrm{Ra}$ and the rate of glucose infusion. The hepatic $\left[{ }^{14} \mathrm{C}\right]$-PEP and $\left[{ }^{3} \mathrm{H}\right] /\left[{ }^{14} \mathrm{C}\right]$-UDP-glucose-specific activities were measured by HPLC, and the rates of PEP-gluconeogenesis (GNG) were calculated. The percentage of the hepatic glucose-6-phos- phate pool directly derived from plasma glucose (direct pathway) was calculated as the ratio of liver $\left[{ }^{3} \mathrm{H}\right]$-UDP-glucose and plasma $\left[{ }^{3} \mathrm{H}-3\right]$-glucose-specific activities. Gluconeogenesis was estimated from the specific activities of $\left[{ }^{14} \mathrm{C}\right]$-labeled hepatic UDP-glucose (assumed to reflect the specific activity of hepatic glucose-6phosphate) and hepatic PEP after the infusion of [U- $\left.{ }^{14} \mathrm{C}\right]-\mathrm{lac}-$ tate and $\left[{ }^{3} \mathrm{H}-3\right]$-glucose by application of the following formula: $\mathrm{GNG}=\mathrm{TGO} \times\left[{ }^{14} \mathrm{C}\right]-\mathrm{UDP}$-glucose SA $/\left[{ }^{14} \mathrm{C}\right]-\mathrm{PEP} \mathrm{SA} \times 2$, where SA is the specific activity, and TGO is the total glucose output.

Western blot analyses. Liver tissues were homogenized in $20 \mathrm{mM}$ MOPS, 2 mM EGTA, 5 mM EDTA, $30 \mathrm{mM}$ sodium fluoride, 40 $\mathrm{mM} \beta$-glycerophosphate, $10 \mathrm{mM}$ sodium pyrophosphate, $2 \mathrm{mM}$ orthovanadate, $0.5 \%$ NP-40, Complete phosphatase inhibitor cocktail (Roche). Protein concentration was measured by use of a BCA protein quantification kit (Pierce, Rockford, Illinois, USA). Extracts were fractionated by use of either a $4 \%$ to $12 \%$ BisTris NuPAGE (Invitrogen) or Criterion XT (Bio-Rad Laboratories Inc.) gel and were blotted as described (39). Primary antibodies against the phosphorylated and unphosphorylated $\alpha$ subunit of AMPK, phospho-Akt473, Akt, and phospho-GSK3 (Cell Signaling Technology Inc., Beverly, Massachusetts, USA), and goat anti-rabbit HRP-conjugated secondary antibodies (Santa Cruz Biotechnology Inc., Santa Cruz, California, USA) were used with ECL reagents (PerkinElmer). Binding was imaged on Kodak MS films and quantified by application of ImageQuant software (Amersham-Pharmacia).

All values are presented as the mean \pm SE. Comparisons among groups were made by application of analysis of variance followed by unpaired, nonparametric Student's $t$ test. Differences were considered statistically significant at $P<0.05$. The study protocol was reviewed and approved by the Institutional Animal Care and Use Committee of the Albert Einstein College of Medicine.

\section{Acknowledgments}

This work was supported by grants from the NIH (R01-DK45024 and ROI-DK48321 to L. Rossetti and R01-DK55758 to P.E. Scherer), the American Diabetes Association (to L. Rossetti and P.E. Scherer), and the Core laboratories of the Albert Einstein Diabetes Research and Training Center (P60-DK 20541).

Received for publication February 6, 2004, and accepted in revised form May 25, 2004.

Address correspondence to: Luciano Rossetti, Department of Pharmacology and Diabetes Research \& Training Center, Albert Einstein College of Medicine, 1300 Morris Park Avenue, New York, New York 10461, USA. Phone: (718) 430-4118; Fax: (718) 430-8557; E-mail: rossetti@aecom.yu.edu.
1. Kahn, B.B., and Flier, J.S. 2000. Obesity and insulin resistance. J. Clin. Invest. 106:473-481.

2. Kopelman, P.G., and Hitman, G.A. 1998. Diabetes. Exploding type II [review]. Lancet. 352(Suppl. 4): SIV5.

3. Porte, D., Jr., et al. 1998. Obesity, diabetes and the central nervous system. Diabetologia. 41:863-881.

4. Flegal, K.M., Carroll, M.D., Ogden, C.L., and Johnson, C.L. 2002. Prevalence and trends in obesity among US adults, 1999-2000. JAMA. 288:1723-1727.

5. Ogden, C.L., Flegal, K.M., Carroll, M.D., and Johnson, C.L. 2002. Prevalence and trends in overweight among US children and adolescents, 1999-2000. JAMA. 288:1728-1732.
6. Halaas, J.L., et al. 1995. Weight-reducing effects of the plasma protein encoded by the obese gene. Science. 269:543-546.

7. Halaas, J.L., et al. 1997. Physiological response to long-term peripheral and central leptin infusion in lean and obese mice. Proc. Natl. Acad. Sci. U. S. A 94:8878-8883.

8. Steppan, C.M., et al. 2001. A family of tissue-specific resistin-like molecules. Proc. Natl. Acad. Sci. U. S. A. 98:502-506.

9. Steppan, C.M., et al. 2001. The hormone resistin links obesity to diabetes. Nature. 409:307-312.

10. Rajala, M.W., Obici, S., Scherer, P.E., and Rossetti, L. 2003. Adipose-derived resistin and gut-derived resistin-like molecule-beta selectively impair insulin action on glucose production. J. Clin. Invest. 111:225-230. doi:10.1172/JCI200316521.

11. Clore, J.N., Helm, S.T., and Blackard, W.G. 1995. Loss of hepatic autoregulation after carbohydrate overfeeding in normal man. J. Clin. Invest. 96:1967-1972.

12. Oakes, N.D., Cooney, G.J., Camilleri, S., Chisholm, D.J., and Kraegen, E.W. 1997. Mechanisms of liver and muscle insulin resistance induced by chronic high-fat feeding. Diabetes. 46:1768-1774.

13. Wang, J., et al. 2001. Overfeeding rapidly induces leptin and insulin resistance. Diabetes. 50:2786-2791.

14. Boden, G., Chen, X., Ruiz, J., White, J.V., and Rossetti, L. 1994. Mechanisms of fatty acid-induced 
inhibition of glucose uptake. J. Clin. Invest. 93:2438-2446.

15. Bergman, R.N., et al. 2001. Central role of the adipocyte in the metabolic syndrome. J. Investig. Med. 49:119-126.

16. Sindelar, D.K., et al. 1997. The role of fatty acids in mediating the effects of peripheral insulin on hepatic glucose production in the conscious dog. Diabetes. 46:187-196.

17. Leroy, P., et al. 1996. Expression of ob gene in adipose cells. Regulation by insulin. J. Biol. Chem. 271:2365-2368.

18. Soukas, A., Cohen, P., Socci, N.D., and Friedman, J.M. 2000. Leptin-specific patterns of gene expression in white adipose tissue. Genes Dev. 14:963-980.

19. Berg, A.H., Combs, T.P., Du, X., Brownlee, M., and Scherer, P.E. 2001. The adipocyte-secreted protein Acrp30 enhances hepatic insulin action. Nat. Med. 7:947-953.

20. Olefsky, J.M., and Saltiel, A.R. 2000. PPAR gamma and the treatment of insulin resistance. Trends Endocrinol. Metab. 11:362-368.

21. Song, H., et al. 2002. Resistin is regulated by C/ EBPs, PPARs, and signal-transducing molecules. Biochem. Biophys. Res. Commun. 299:291-298.

22. Fukui, Y., and Motojima, K. 2002. Expression of resistin in the adipose tissue is modulated by various factors including peroxisome proliferator-activated receptor alpha. Diabetes Obes. Metab. 4:342-345.

23. Combs, T.P., et al. 2002. Induction of adipocyte complement-related protein of 30 kilodaltons by PPARgamma agonists: a potential mechanism of insulin sensitization. Endocrinology. 143:998-1007. 24. McTernan, P.G., et al. 2002. Increased resistin gene and protein expression in human abdominal adipose tissue. J. Clin. Endocrinol. Metab. 87:2407.

25. Steppan, C.M., and Lazar, M.A. 2002. Resistin and obesity-associated insulin resistance. Trends Endocrinol. Metab. 13:18-23.

26. Yamauchi, T., et al. 2002. Adiponectin stimulates glucose utilization and fatty-acid oxidation by activating AMP-activated protein kinase. Nat. Med. 8:1288-1295

27. Barthel, A., Schmoll, D., Kruger, K.D., Roth, R.A., and Joost, H.G. 2002. Regulation of the forkhead transcription factor FKHR (FOXO1a) by glucose starvation and AICAR, an activator of AMP-activated protein kinase. Endocrinology. 143:3183-3186.

28. Combs, T.P., Berg, A.H., Obici, S., Scherer, P.E., and Rossetti, L. 2001. Endogenous glucose production is inhibited by the adipose-derived protein Acrp30. J. Clin. Invest. 108:1875-1881. doi:10.1172/ JCI200114120.

29. Minokoshi, Y., et al. 2002. Leptin stimulates fattyacid oxidation by activating AMP-activated protein kinase. Nature. 415:339-343.

30. Patel, L., et al. 2003. Resistin is expressed in human macrophages and directly regulated by PPAR gamma activators. Biochem. Biophys. Res. Commun. 300:472-476.

31. Degawa-Yamauchi, M., et al. 2003. Serum resistin (FIZZ3) protein is increased in obese humans. J. Clin. Endocrinol. Metab. 88:5452-5455.

32. Azuma, K., et al. 2003. Correlation between serum resistin level and adiposity in obese individuals. Obes. Res. 11:997-1001.

33. Hartman, H.B., Hu, X., Tyler, K.X., Dalal, C.K., and Lazar, M.A. 2002. Mechanisms regulating adipocyte expression of resistin. J. Biol. Chem. 277:19754-19761.

34. Shojima, N., et al. 2002. Humoral regulation of resistin expression in 3T3-L1 and mouse adipose cells. Diabetes. 51:1737-1744.

35. Xu, H., et al. 2003. Chronic inflammation in fat plays a crucial role in the development of obesity-related insulin resistance. J. Clin. Invest. 112:1821-1830. doi:10.1172/JCI200319451.

36. Rossetti, L., et al. 1997. Peripheral but not hepatic insulin resistance in mice with one disrupted allele of the glucose transporter type 4 (GLUT4) gene. J. Clin. Invest. 100:1831-1839.

37. Xu, Y., Jones, B.E., Neufeld, D.S., and Czaja, M.J. 1998. Glutathione modulates rat and mouse hepatocyte sensitivity to tumor necrosis factor toxicity. Gastroenterology. 115:1229-1237.

38. Baker, B.F., et al. 1997. 2'-O-(2-methoxy)ethylmodified anti-intercellular adhesion molecule 1 (ICAM-1) oligonucleotides selectively increase the ICAM-1 mRNA level and inhibit formation of the ICAM-1 translation initiation complex in human umbilical vein endothelial cells. J. Biol. Chem. 272:11994-12000.

39. Rajala, M.W., et al. 2002. Cell type-specific expression and coregulation of murine resistin and resistin-like molecule-alpha in adipose tissue. Mol. Endocrinol. 16:1920-1930. 